\title{
Per-operative stent placement in the right pulmonary artery; a hybrid technique for the management of pulmonary artery branch stenosis at the time of pulmonary valve replacement in adult Fallot patients
}

\author{
F. Windhausen • S. M. Boekholdt • B. J. Bouma • \\ M. Groenink • A. P. C. M. Backx • R. J. de Winter • \\ B. J. M. Mulder • M. G. Hazekamp • D. R. Koolbergen
}

Published online: 14 September 2011

(C) The Author(s) 2011. This article is published with open access at Springerlink.com

\begin{abstract}
After having undergone surgical correction at an early age, many patients with tetralogy of Fallot develop long-term complications including progressive pulmonary regurgitation and peripheral pulmonary stenosis. A high percentage of these patients need to undergo a second operation in their adolescence or early adulthood. If simultaneous treatment of both pulmonary regurgitation and peripheral pulmonary stenosis is warranted, a complete surgical approach has several disadvantages. We describe four cases of Fallot patients with severe pulmonary regurgitation and peripheral pulmonary stenosis who were treated using a hybrid approach involving surgical implantation of a pulmonary homograft and peroperative stenting of the pulmonary artery.
\end{abstract}

F. Windhausen - S. M. Boekholdt - B. J. Bouma - M. Groenink

R. J. de Winter · B. J. M. Mulder $(\bowtie)$

Department of Cardiology, Academic Medical Center,

PO Box 22660, 1100 DD Amsterdam, Netherlands

e-mail: b.j.mulder@amc.nl

A. P. C. M. Backx

Department of Pediatric Cardiology, Academic Medical Center,

Amsterdam, Netherlands

M. G. Hazekamp • D. R. Koolbergen

Department of Pediatric Cardiac Surgery,

Academic Medical Center,

Amsterdam, Netherlands

M. G. Hazekamp • D. R. Koolbergen

Amsterdam and Department of Cardiothoracic Surgery,

Leiden University Medical Center,

Leiden, Netherlands
Keywords Tetralogy of Fallot · Pulmonary homograft . Pulmonary artery stenting

During the first decennia of cardiac surgery (1960s and 1970s), neonates born with tetralogy of Fallot were typically treated at a young age with a surgical shunt between the aorta and pulmonary artery to increase pulmonary blood flow and thus oxygenation, as a bridge to complete correction. A technique frequently used at that time was a direct anastomosis between the ascending aorta and the right PA at the side where they cross, a so-called Waterston shunt. Complete surgical correction was then performed in the first years of life and involved taking down the shunt, closure of the ventricular septum defect and relief of the pulmonary valve stenosis with or without a transannular patch $[1,2]$.

The most common long-term complication after complete surgical correction of tetralogy of Fallot is progressive pulmonary regurgitation. Pulmonary valve replacement is indicated when patients become symptomatic and/or in case of progressive dilatation and dysfunction of the right ventricle. [3] Another long-term complication is the development of peripheral stenosis of the right pulmonary artery at the previous anastomosis site of the Waterston shunt. Frequently, the severity of pulmonary regurgitation and its deleterious long-term effects are augmented by coexisting pulmonary artery stenosis and should therefore be addressed at the time of surgery.

Pulmonary valve replacement with a homograft is a lowrisk procedure which can usually be performed on a beating heart with a short cardiopulmonary bypass (CPB) run. 
Simultaneous treatment of peripheral pulmonary artery stenosis, especially when due to a previous Waterston shunt, is a more challenging surgical task, with disappointing long-term results [4]. The surgical approach requires aortic clamping, cardioplegia and often transsection of the aorta, which is associated with additional periprocedural risk. Even if a good initial result is achieved, any patchplasty of the right PA branch is immediately compromised by the, often mildly dilated, ascending aorta and by scarring in the long term.

In this report we present four tetralogy of Fallot patients with severe pulmonary regurgitation and peripheral stenosis of the right branch of the pulmonary artery who underwent a hybrid strategy with surgical pulmonary valve replacement with a homograft and peroperative stenting to treat the peripheral pulmonary artery stenosis.

\section{Patients}

At neonatal age, all patients were palliated with a Waterston shunt to increase pulmonary blood flow. When they were toddlers, the shunt was taken down and total surgical repair was performed with enlargement of the hypoplastic pulmonary trunk using a transannular patch (except in Case $\mathrm{C}$ where no transannular patch was used). All patients gradually developed severe pulmonary regurgitation and progressive right ventricular dilatation and dysfunction, as well as a significant stenosis of the right pulmonary artery at the anastomosis site of the former Waterston shunt.

Case A was a male patient of 39 years who was transferred from a referring hospital for management of severe pulmonary regurgitation and sustained ventricular tachycardia with a left bundle branch morphology and an inferior axis. He was known to have severe pulmonary regurgitation but had been asymptomatic until admission. Echocardiography showed severe pulmonary regurgitation and a dilated right ventricle with preserved systolic function.

Case B was a 38-year-old female who had visited our outpatient clinic on a regular basis since early childhood. At the age of 14 she was diagnosed with moderate pulmonary valve stenosis and regurgitation. The right ventricular pressure at that time was $70 / 12 \mathrm{mmHg}$ as a consequence of stenoses in both the right and left pulmonary artery branches (gradient $20 \mathrm{mmHg}$ and $35 \mathrm{mmHg}$, respectively) in combination with moderate pulmonary valve stenosis. She had been completely asymptomatic and was managed conservatively. At the age of 31 , pulmonary regurgitation had become severe but there were still no echocardiographic signs of dilatation or dysfunction of the right ventricle. At the age of 39, cardiac MRI showed a significant increase of right ventricular end-diastolic and end-systolic volumes within one year and confirmed severe pulmonary regurgitation.
Case $\mathrm{C}$ was a female patient with tetralogy of Fallot who had given birth to twins without any cardiac complications. She visited the outpatient clinic on a regular basis because of moderate pulmonary regurgitation with normal dimensions and function of the right ventricle. She was asymptomatic until the age of 36 . She gradually developed right ventricular systolic dysfunction due to severe pulmonary regurgitation in combination with pulmonary valve stenosis as well as stenosis of the right and left pulmonary artery branches. Her exercise capacity gradually deteriorated and she developed signs of right-sided heart failure.

Case D was a female tetralogy of Fallot patient who had undergone total correction at the age of 5. At the age of 12, she had developed severe stenosis of the right branch of the pulmonary artery (gradient $75 \mathrm{mmHg}$ ) which was treated with surgical patch-plasty. She remained asymptomatic until the age of 37 . At the age of 37 her exercise capacity deteriorated. MRI showed progression of right ventricular dilatation, impaired right ventricular systolic function, severe pulmonary regurgitation, an aneurysmatic right ventricular outflow tract (RVOT) and severe ostial stenosis of the right pulmonary artery branch.

\section{The hybrid procedures}

The hybrid procedure combines surgical and interventional techniques. All procedures were performed on CPB. The pulmonary trunk was incised longitudinally and the pulmonary artery branches were visualised directly. The length of the stent was selected on the basis of the vessel diameter as visualised by preoperative imaging (angiography and MRI in particular), the location of the stenosis, and peroperative anatomical findings. The CPZ8 stent allows expansion from $8.0 \mathrm{~mm}$ to $24.0 \mathrm{~mm}$. The CPZ8 stent was hand-crimped on a SHYTAK II balloon and placed across the lesion by the interventional cardiologist. The stent was inflated according to manufacturer's guidelines. Positioning of the stent was done under direct vision of the proximal part of the stent and the distal aspect was palpated digitally to ensure that the stent would not compromise the ostium of the right upper lobe branch.

In all patients, a pulmonary homograft was used to replace the pulmonary valve and pulmonary trunk. Additional reconstruction of the aneurysmatic RVOT was performed in Cases A, B and D.

In Case A, the procedure was performed on a beating heart. Predilatation was performed using a $12 \mathrm{~mm}$ balloon (6 atm) using an Amplatzer stiff guidewire. The length of the CPZ8 stent was $28 \mathrm{~mm}$; after balloon inflation the diameter of the stent was $15 \mathrm{~mm}$ (4 atm). The CPB time was $136 \mathrm{~min}$.

In Case B, predilatation was performed using a $12 \mathrm{~mm}$ balloon (6 atm) using an Amplatzer stiff guidewire. The length of the CPZ8 stent was $22 \mathrm{~mm}$, after balloon inflation 
the diameter of the stent was $14 \mathrm{~mm}(4 \mathrm{~atm})$. The proximal part of the stent was post-dilated to $18 \mathrm{~mm}$ to assure good fit. The CPB time was 85 min.

In Case C, a CPZ8 stent (length $34 \mathrm{~mm}$ ) was positioned across the severely stenotic proximal right pulmonary artery. After balloon inflation (4 atm) the diameter of the stent was $15 \mathrm{~mm}$. The position of the stent was good, but waisting of the stent was seen in the middle section of the stent. Therefore the stent was post-dilated $(8 \mathrm{~atm})$. The procedure was complicated by a tear of the right pulmonary artery at the distal end of the stent for which aortic clamping was necessary for $25 \mathrm{~min}$. The right pulmonary artery was repaired successfully using a triangular patch. The proximal left pulmonary artery stenosis was repaired with the use of the homograft. The CPB time was $146 \mathrm{~min}$.

In Case D, femoral cannulation was necessary because of severe adhesions as a consequence of previous operations. Aortic clamping of $47 \mathrm{~min}$ was necessary because of excessive collateral flow and for optimal exposition of the right pulmonary artery ostium. The length of the $\mathrm{CP}$ stent was $22 \mathrm{~mm}$, after balloon inflation (4 atm) the diameter of the stent was $12 \mathrm{~mm}$. The CPB time was $147 \mathrm{~min}$.

\section{Follow-up}

In all patients, a dramatic improvement of right ventricular systolic function was observed, allowing weaning from inotropic support and artificial ventilation within a few hours after the procedure. Postoperative echocardiography showed mild pulmonary regurgitation and an improvement of right ventricular dimensions and function in all patients. After 3 years of follow-up, Case A, B and C patients are still asymptomatic. Case $\mathrm{D}$ is also asymptomatic but follow-up is only 2 months.

\section{Discussion}

In all cases, the stenosis of the right pulmonary artery was located at the site of the former Waterston shunt. Soon after the introduction of this surgical technique it was recognised that the Waterston shunt was associated with problems such as kinking of the pulmonary artery, pulmonary overflow with congestive heart failure and eventually pulmonary vascular disease. Take-down of the shunt was performed by direct suturing from inside the aorta, simple ligation or detachment and patch-plasty of the right pulmonary artery branch. Irrespective of the surgical technique used, there is a high incidence of severe obstruction of the right pulmonary artery due to scarring in combination with compression by the ascending aorta. [4] Due to the short-term and long-term complications associated with the Waterston shunt, this technique was abandoned and other shunt techniques were introduced, such as the modified Blalock-Taussig shunt. Over the past two decades, further developments in surgical techniques and perioperative care have led to a tendency towards primary correction at young age.

Nowadays in our centre, primary correction is performed between 3 and 6 months and a shunt is avoided whenever possible. Only in case of severe hypoplasia of the PA branches and/or tree or in case of unfavourable RVOT anatomy (a long and severely obstructive infundibulum requiring a long transannular patch) is an initial modified Blalock-Taussig shunt still used.

Percutaneous stenting of the pulmonary artery was introduced in 1991 [5]. Subsequent follow-up studies demonstrated that stents maintain long-term vessel patency and can be dilated further at subsequent catheterisation. [69] Percutaneous stenting is now an established treatment modality for older children and adults with branch pulmonary artery stenosis $[10,11]$, although high rates of early complications are reported [12].

Intra-operative placement of stents under direct vision as a hybrid procedure has nowadays completed the spectrum of pulmonary artery stenting. A hybrid procedure shortens $\mathrm{CPB}$ time and avoids the need for hypothermia and transsection of the ascending aorta and for patch-plasty of the right pulmonary artery. Furthermore, complications of percutaneous stent placement, such as haemodynamic instability and vascular tear, can be avoided or easily addressed in the operative setting with $\mathrm{CPB}$, thereby making the procedure safe and effective. [13-18]

Close collaboration between cardiac surgeons, imaging and interventional cardiologists is essential in the modern management of congenital heart defects. The hybrid approach described above is an example of how this can be accomplished. In our CAHAL collaboration between Amsterdam Medical Centre, Leiden University Medical Centre and the VU University Medical Centre, the hybrid procedure was shown to be a safe and effective treatment option for patients with severe pulmonary regurgitation and stenosis of the pulmonary arteries. Long-term follow-up of stented patients will be necessary to establish the long-term impact of this hybrid procedure.

Open Access This article is distributed under the terms of the Creative Commons Attribution Noncommercial License which permits any noncommercial use, distribution, and reproduction in any medium, provided the original author(s) and source are credited.

\section{References}

1. Waterston DJ. Treatment of Fallot's tetralogy in children under one year of age. Rozhl Chir. 1962;41:181. 
2. Murphy JG, Gersh BJ, Mair DD, et al. Long-term outcome in patients undergoing surgical repair of tetralogy of Fallot. N Engl J Med. 1993;329:593-9.

3. Oosterhof T, van Straten A, Vliegen HW, et al. Preoperative thresholds for pulmonary valve replacement in patients with corrected tetralogy of Fallot using cardiovascular magnetic resonance. Circulation. 2007;116:545-51.

4. Wilson JM, Mack JW, Turley K, et al. Persistent stenosis and deformity of the right pulmonary artery after correction of the Waterston anastomosis. J Thorac Cardiovasc Surg. 1981;82 (2):169-75.

5. O'Laughlin MP, Perry SB, Lock JE, et al. Use of endovascular stents in congenital heart disease. Circulation. 1991;83:1923-39.

6. O'Laughlin MP, Slack MC, Grifka RG, et al. Implantation and intermediate-term follow-up of stents in congenital heart disease. Circulation. 1993;88:605-14.

7. Shaffer KM, Mullins CE, Grifka RG, et al. Intravascular stents in congenital heart disease: Short- and long-term results from a large single-center experience. J Am Coll Cardiol. 1998;31:661-7.

8. Ing FF, Grifka RG, Nihill MR, et al. Repeat dilation of intravascular stents in congenital heart defects. Circulation. 1995;92:893-7.

9. McMahon CJ, El-Said HG, Grifka RG, et al. Redilation of endovascular stents in congenital heart disease: Factors implicated in the development of restenosis and neointimal proliferation. J Am Coll Cardiol. 2001;38:521-6.
10. Fogelman R, Nykanen D, Smallhorn JF, et al. Endovascular stents in the pulmonary circulation. Clinical impact on management and medium-term follow-up. Circulation. 1995;92(4):881-5.

11. Bergersen L, Lock JE. What is the current option of first choice for treatment of pulmonary arterial stenosis? Cardiol Young. 2006;16(4):329-38.

12. van Gameren M, Witsenburg M, Takkenberg JJ, et al. Early complications of stenting in patients with congenital heart disease: a multicentre study. Eur Heart J. 2006;27(22):2709-15.

13. Hjortdal VE, Redington AN, de Leval MR, et al. Hybrid approaches to complex congenital cardiac surgery. Eur J Cardiothorac Surg. 2002;22:885-90.

14. Coles JG, Yemets I, Najm HK, et al. Experience with repair of congenital heart defects using adjunctive endovascular devices. J Thorac Cardiovasc Surg. 1995;110:1513-20.

15. Peters B, Ewert P, Berger F. The role of stents in the treatment of congenital heart disease: Current status and future perspectives. Ann Pediatr Card. 2009;2:3-23.

16. Schmitz C, Esmailzadeh B, Herberg U, et al. Hybrid procedures can reduce the risk of congenital cardiovascular surgery. Eur $\mathrm{J}$ Cardiothorac Surg. 2008;34(4):718-25.

17. Bokenkamp R, Blom NA, de Wolf D, et al. Intraoperative stenting of pulmonary arteries. Eur J Cardiothorac Surg. 2005;27(4):544-7.

18. Menon SC, Cetta F, Dearani JA, et al. Hybrid intraoperative pulmonary artery stent placement for congenital heart disease. Am J Cardiol. 2008;102(12):1737-41. 